\title{
Dynamical properties and plasmon dispersion of a weakly degenerate correlated one-component plasma
}

\author{
V. Golubnychiy ${ }^{1}$, M. Bonitz ${ }^{1}$, D. Kremp ${ }^{1}$, and M. Schlanges ${ }^{2}$ \\ ${ }^{1}$ Fachbereich Physik, Universität Rostock, Universitätsplatz 3, 18051 Rostock, FRG \\ ${ }^{2}$ Institut für Physik, Ernst-Moritz-Arndt Universität Greifswald, Domstr. 10a, 17489 \\ Greifswald, FRG
}

\begin{abstract}
Classical Molecular Dynamics (MD) simulations for a one-component plasma (OCP) are presented. Quantum effects are included in the form of the Kelbg potential. Results for the dynamical structure factor are compared with the Vlasov and RPA (random phase approximation) theories. The influence of the coupling parameter $\Gamma$, degeneracy parameter $\rho \Lambda^{3}$ and the form of the pair interaction on the optical plasmon dispersion is investigated. An improved analytical approximation for the dispersion of Langmuir waves is presented.
\end{abstract}

Typeset using REVTEX 


\section{INTRODUCTION}

The model of a classical one-component plasma has - due to its simplicity - been widely investigated both theoretically and with various numerical and simulation methods, see e.g. [1] 3] and [4,5], respectively. Since the pioneering numerical work of Brush, Sahlin, Teller [6], the thermodynamic and dynamic characteristics of the classical OCP have been studied in detail. In particular, the dependence of the properties on the coupling parameter $\Gamma=4 \pi e^{2} /\left(\bar{r} k_{B} T\right)$, where $\bar{r}=\left(\frac{3}{4 \pi \rho}\right)^{1 / 3}$ is the mean interparticle distance and $\rho$ the density, have been investigated up to very large values of $\Gamma[0,8]$. Among the most important thermodynamic results is the prediction of crystallization at values of $\Gamma$ of the order of $172-180$ [9:10]. Furthermore, investigations of the dynamic properties of strongly correlated classical plasmas have indicated that the wave number dependent plasmon dispersion changes from monotonic growth, common for weakly coupled plasmas, to a decreasing dispersion around $\Gamma \approx 3 \llbracket \mathbb{1}$.

On the other hand, there is growing interest in the dynamic properties of dense quantum plasmas, in particular in astrophysics, laser plasmas and condensed matter. While the case of strong degeneracy (strong quantum limit) and weak coupling at very high densities is well described by the random phase approximation (RPA, see e.g. [11]14]), the properties at intermediate coupling and degeneracy remain poorly explored. Especially, one is interested in the dynamic plasma behavior in cases where the average kinetic energy is of the same order as the mean potential energy, i.e. $\Gamma \sim 1$, where collisionless theories such as the RPA fail, e.g. 12,13,15]. For these situations, quantum molecular dynamics (QMD) simulations [16] are the appropriate numerical approach which, however, is yet lacking the required efficiency. For weakly degenerate plasmas, with $\rho \Lambda^{3} \leq 1$, where $\Lambda$ is the DeBroglie wave length (see below), it is expected that one can perform much simpler classical MD simulations using effective quantum pair potentials, e.g. [3, 17]. These potentials can be derived from the 2particle Slater sum using Morita's method. It is the aim of this paper to explore this MD approach in detail, especially for the analysis of the optical (Langmuir) plasmon dispersion. 
It is natural to start this analysis with OCP-simulations because they have the advantage of the absence of a collapse of oppositely charged particles at small distances. On the other hand, the existence of a homogeneous background of oppositely charged particles leads to some additional technical difficulties compared to 2-component systems, due to restricted carrier rearrangement causing less effective screening of the Coulomb interaction. One major problem of MD simulations of dynamical properties is that the behavior at small wave numbers is difficult to investigate. The reason is that large box-sizes are required which, for the analysis of high density plasmas, translates into large particle numbers. The current increase of available computer power gives one the possibility to investigate size-dependent properties like the density-density correlations $\left\langle\rho_{\vec{k}}(0) \rho_{-\vec{k}}(t)\right\rangle$ for smaller k-vectors than before. In this paper, we are able to present accurate results for the dynamical properties of the OCP, such as the dynamical structure factor and the wave vector dispersion of Langmuir oscillations. Our simulations for intermediate values of the coupling parameter, $\Gamma=1 \ldots 4$, show an interesting dispersion: the frequency increases up to a maximum and, for large wave numbers, decreases again. Further, we investigate the role of quantum effects by comparing simulations with the Coulomb potential and an effective quantum pair potential (Kelbg potential [17]) for the region of small and intermediate coupling. We found that quantum diffraction effects have noticable influence on the behavior of the optical dispersion curves. Increase of the degeneracy leads to a softenig of the dispersion $\omega(k)$, especially at intermediate wave vectors.

\section{DYNAMICAL PROPERTIES OF THE OCP}

\section{A. Statistical approach}

A central quantity to determine the dynamic properties of charged many-particle systems is the frequency-dependent dielectric function $\epsilon(\vec{k}, \omega)$ which, for the OCP, is given by

$$
\epsilon(\vec{k}, \omega)=1-U_{C}(\vec{k}) \Pi(\vec{k}, \omega)
$$


Here $U_{C}(\vec{k})$ is the spatial Fourier transform of the Coulomb potential, $U_{C}(k)=4 \pi e^{2} / k^{2}$, and $\Pi(\vec{k}, \omega)$ is the longitudinal polarization function. Thus, many-body effects enter the dielectric function via $\Pi$. There exist many approximations for the latter function, the simplest one being mean-field theories which neglect short-range correlation effects, i.e. collisions between the particles. For the classical OCP, the mean-field result is the Vlasov polarization:

$$
\Pi_{\mathrm{Vlasov}}(\vec{k}, \omega)=-\frac{1}{m} \int \frac{d^{3} v}{(2 \pi)^{3}} \frac{\vec{k}}{\omega-\vec{k} \vec{v}+i \delta} \frac{\partial F(\vec{v})}{\partial \vec{v}} .
$$

Here $\delta \rightarrow+0$, indicating the retarded (causal) character of the polarization and the dielectric function. Further, $F$ is the distribution function. The Vlasov polarization applies only to classical plasmas, where the wave character of the particles can be neglected. Quantum effects are important if the interparticle distance or the Debye radius become comparable to the DeBroglie wave length $\Lambda=h / \sqrt{2 \pi m k_{B} T}$. Therefore, quantum diffraction effects should show up in the dielectric properties at large wave numbers. The quantum generalization of the Vlasov polarization is the RPA polarization function given by

$$
\Pi_{R P A}(\vec{k}, \omega)=-\int \frac{d^{3} p}{(2 \pi \hbar)^{3}} \frac{f(\vec{p})-f(\vec{p}-\hbar \vec{k})}{\hbar \omega+\frac{p^{2}}{2 m}-\frac{(\vec{p}+\hbar \vec{k})^{2}}{2 m}+i \delta}
$$

In this paper we consider only plasmas in equilibrium, so $F$ and $f$ are the Maxwell and Fermi function, respectively. One readily confirms that, in the limit of long wavelengths, $\vec{k} \rightarrow 0$, indeed the RPA result (3) goes over to the Vlasov polarization function (2). An important quantity which follows from dielectric function (11) via the fluctuation-dissipation theorem is the dynamical structure factor $S(\vec{k}, \omega)$

$$
S(\vec{k}, \omega)=-\frac{k_{B} T}{\pi U_{C}(k) \omega} \operatorname{Im} \frac{1}{\epsilon(\vec{k}, \omega)}
$$

which shows the frequency spectrum of density fluctuations for a given value of $\vec{k}$.

As mentioned above, the mean field expressions (2) and (3) neglect short-range correlations and are, therefore, valid only for weakly coupled plasmas, $\Gamma \ll 1$. There exist many theoretical concepts to go beyond the RPA which are based on quantum kinetic theory, density functional theory and other approaches. This is beyond the scope of this paper, see 
e.g. Ref. [12,13,15] and references therein. Here, we consider the alternative approach to the OCP at finite coupling which is based on molecular dynamics simulations.

\section{B. Molecular dynamics approach to the dynamical properties}

The dielectric and dynamical properties of an interacting many-particle system are easily accessible from the density-density correlation function which is defined as

$$
A(\vec{k}, t)=\frac{1}{N}\left\langle\rho_{\vec{k}}(t) \rho_{-\vec{k}}(0)\right\rangle,
$$

where $N$ is the number of particles. $\rho_{\vec{k}}(t)$ is the Fourier component of the density,

$$
\rho_{\vec{k}}(t)=\sum_{i=1}^{N} e^{i \vec{k} \vec{r}_{i}(t)},
$$

which is computed from the trajectories $\vec{r}_{i}(t)$ of all particles. The dynamical structure factor is just the Fourier transform of the density-density correlation function (5)

$$
S(\vec{k}, \omega)=\frac{1}{2 \pi} \int_{-\infty}^{+\infty} d t e^{i \omega t} A(\vec{k}, t) .
$$

Equation (77) can be directly compared to formula (14) and, thus, allows for a comparison of the simulation results with the statistical theories. Furthermore, Eq. (7) allows to investigate the influence of quantum effects on the dynamical properties and plasmon dispersion of the OCP. Variations of the interaction potential (see below) directly affect the particle trajectories and, via Eqs. (5)-(7), the dynamical structure factor.

\section{DETAILS OF THE MD-SIMULATIONS}

The simulations have been performed in a cube of length $L$ containing $N$ interacting electrons on a uniform positive background. For this system, we solved Newton's equations of motion containing all pair interactions which are derived from a total potential $U(r)$, see below. As an algorithm of motion we used a second-order scheme in form of the Swope algorithm [18]. Since our simulations are performed in the microcanocial ensemble, the mean 
kinetic energy may change. Therefore, to maintain the chosen value of temperature and $\Gamma$, we applied scaling (renormalization) of all velocities at every second step.

A central goal of our simulations was to study the influence of quantum effects. We, therefore, performed several simulations which used either a Coulomb potential or an effective quantum pair potential (see below). To permit flexibility in the use of the potential, $U$ was divided into a short-range and a long-range part, $U=U^{\mathrm{sr}}+U^{\mathrm{lr}}$, where quantum effects influence only $U^{\text {sr }}$, whereas the behavior at large distances, $U^{\mathrm{lr}}$, is dominated by the long-range Coulomb interaction. Let us first describe the treatment of the long-range term.

\section{A. Long-range interaction}

The long-range interaction was computed in standard way using periodic boundary conditions and the Ewald summation procedure [20,21]. As a result, the long-range potential is given by the Coulomb interaction in the main box and all image cells:

$$
\begin{aligned}
U^{\mathrm{lr}}(\vec{r}) & =4 \pi e^{2} \sum_{i \neq j}^{N} V_{\text {Ewald }}\left(\vec{r}_{i j}\right), \\
V_{\text {Ewald }}(\vec{r}) & =\sum_{\vec{n}=0}^{n_{x}, n_{y}, n_{z} \leq 1} \frac{\operatorname{erfc}[\sqrt{\pi}|(\vec{r}+\vec{n} L) / L|]}{|\vec{r}+\vec{n} L|}+\sum_{\vec{n} \neq 0}^{n_{x}, n_{y}, n_{z} \leq 5, n^{2} \leq 27} \frac{\exp \left(-\pi n^{2}\right) \cos (2 \pi \vec{n} \vec{r} / L)}{\pi n^{2} L}-\frac{1}{L},
\end{aligned}
$$

where erfc is the complementary error function, $L$ - the side length of the simulation cell and $\vec{n}$ - a vector of integer numbers which labels the periodic images of the simulation box. In this expression, the first term corresponds to a potential of particles with Gaussian broadened charge distribution around the electrons with a width of $\sqrt{\pi}$, the second one the compensating Gaussian distributions, and the last one accounts for the influence of the homogeneous background. It turns out that the second term in (8) can be reduced to 2 loops (one over the particles and one over the vectors $\vec{n}$ in the reciprocal space) and is not very time consuming. The more complicated part is the first term which contains three loops. In case of a two-component plasma, a proper choice of the width of the Gaussian distribution and use of periodic boundary conditions greatly simplifies this term due to cancellations. In contrast, for an OCP, the background cancels the interactions only partially, "statically". As a result, 
convergence of the sum is worse, and one needs to take into account all first neighboring image cells (total of 26) at every time step. The contribution of all neighboring cells except for the main one $(0<|\vec{n}| \leq \sqrt{3})$ was computed, before the start of the simulations and stored in 3-dimensional tables for the potential and forces. During the simulations, we used 3D-bilinear interpolation at every step to obtain the values of the potential and forces for intermediate distances. We found that 100 grid points in every direction are adequate, so the total size of the table was $10^{6}$ elements. The particle interactions inside the main $(\vec{n}=0)$ cell were evaluated directly at every time step without minimum image convention.

\section{B. Short-range interaction. Quantum effects}

Let us now discuss the short-range potential. As has been shown by Kelbg and co-workers [17,19, quantum effects can be treated efficiently by an effective pair potential, the Kelbg potential:

$$
U_{\mathrm{KELBG}}(r, T)=4 \pi e^{2}\left(\frac{1-\exp \left(-r^{2} / \lambda^{2}\right)}{r}+\frac{\sqrt{\pi}}{\lambda} \operatorname{erfc}(r / \lambda)\right)
$$

where $\lambda=\frac{\Lambda}{\sqrt{2 \pi}}$. As a consequence of quantum effects, this potential differs from the Coulomb potential at small distances $r \leq \lambda$ and is finite at $r=0$. Further, it is temperature-dependent via the thermal DeBroglie wavelength. The Kelbg potential can be regarded as the proper quantum pair potential following from the two-particle Slater sum $S_{2}$ without exchange effects:

$$
\ln S_{2}=-\frac{U_{K E L B G}}{k T}+O\left(\Gamma^{2}\right)
$$

It treats quantum diffraction effects exactly, up first order in $\Gamma$. Frequently other quantum pair potentials have been used, including the Deutsch potential [22], which has the same value at $r=0$ but differs from the Kelbg potential at intermediate distances. As was mentioned by Hansen [23], symmetry effects do not have a big influence on the dynamical properties (although they give a major contribution to the static properties, especially for 
the light mass components). Using the Kelbg potential (10), we can immediately separate the short-range part of the interaction,

$$
U^{\mathrm{sr}}(r, T)=4 \pi e^{2}\left(\frac{-\exp \left(-r^{2} / \lambda^{2}\right)}{r}+\frac{\sqrt{\pi}}{\lambda} \operatorname{erfc}(r / \lambda)\right),
$$

which has been calculated together with the first sum of Eq. (91) using the interpolation table.

The Kelbg potential contains just the lowest order quantum corrections (lowest order in $\left.e^{2}\right)$ and is, thus, accurate at small coupling, $\Gamma<1$. Nevertheless, we expect that it correctly reproduces the influence of quantum effects also at intermediate coupling, $\Gamma \leq 5$. Further improvements are straightforward, e.g. by including exchange effects or by evaluating the full two-particle Slater sum. We note that the described numerical procedure applies to such improved quantum pair potentials as well, even if they are not given analytically.

\section{Thermodynamic and dynamical quantities}

Solving Newton's equations with forces derived from the total potential $U^{\mathrm{sr}}+U^{\mathrm{lr}}$, we computed thermodynamic and static quantities, such as total energy and pair distribution function in usual manner. The results will be presented in the next section. Here we discuss some details on computation of the dynamical properties, as they require much more effort and computation time in order to achieve sufficient accuracy.

To obtain useful results for the dynamical structure factor, requires simulation results in a sufficiently broad range of wave numbers and frequencies. Natural units of the wave number and frequency are $1 / \bar{r}$ and the plasma frequency $\omega_{p l}=\sqrt{4 \pi e^{2} \rho / m}$, respectively, which will be used in the following. The minimum wave number $k_{\min }$ depends on the size $L$ of the simulation box and thus, for a given density or coupling parameter, on the number of particles $N$. One readily verifies that $k_{\min }=2 \pi / L=2 \pi(\rho / N)^{1 / 3}$ or, using dimensionless wave numbers, $q_{\min }=k_{\min } \bar{r}=\left(6 \pi^{2} / N\right)^{1 / 3}$. Clearly, to reduce $k_{\min }$ requires an essential increase of the number of particles in the simulation. 
The simulation accuracy can be further increased by taking advantage of the isotropy of the plasma in wave vector space. Indeed, in equilibrium, the density-density correlation function and dynamical structure factor should only depend on the absolute value of the wave vector. On the other hand, the simulations yield slightly different results for different directions of the wave vector. Averaging over all results corresponding to the same absolute value of $\vec{k}$ allows to reduce the statistical error. For example, the minimum wave number $k_{\text {min }}$ corresponds to directions of $\vec{k}$ along either the $\mathrm{x}-, \mathrm{y}$ - or z-axis, cf. Eq. (6), so we can use the average of the three. The next larger value is $\sqrt{2} k_{\text {min }}$, corresponding to the diagonals in the $\mathrm{x}-\mathrm{y}, \mathrm{x}-\mathrm{z}$ and $\mathrm{y}-\mathrm{z}$ planes. The third value, $\sqrt{3} k_{m i n}$, corresponds to the space diagonal and is not degenerate; consequently it carries the largest statistical error. This is the main reason for the fluctuations of the numerical results for the wave vector dispersion, see for example Fig. 5.

Finally, to resolve the collective plasma oscillations, the duration of the simulations has to be much larger than the plasma period. Also, increased simulation times leads to a reduction of the noise. We found that times of the order of 250 plasma periods are adequate.

\section{NUMERICAL RESULTS}

We have performed a series of simulations for varying values of $\Gamma$ and $\rho \Lambda^{3}$, using the Coulomb and Kelbg potential. Also, time step and particle number have been varied until a satisfactory compromise between accuracy and simulation efficiency has been achieved. The parameters of the runs chosen for the figures below are summarized in Table I. We mention that kinetic energy conservation in all runs (if velocity scaling was turned off) did not exceed 0.1\%. Also, the results for the total energy (not shown), in case of the Coulomb potential, agree very well with data from the literature.

We first consider the pair distribution function $g(r)$ for varying interaction potentials and parameter values. Fig. 1 shows $g(r)$ for three values of the coupling parameter, $\Gamma=$ 0.5, 1, 4. As expected, the Coulomb pair distribution function is close to the Debye-Hückel 
limit for small coupling, with increasing $\Gamma$, the deviations, especially around $r=\bar{r}$, grow systematically. The Kelbg pair distributions practically coincide with the Coulomb functions for $r>0.6 \bar{r}$ but deviate from the latter at small distances of the order of the thermal DeBroigle wave length $\Lambda$ where quantum effects are important. Clearly, with increasing degeneracy, the ratio $\Lambda / \bar{r}$ increases, and the deviations extend to larger distances and grow in magnitude. With increasing $\Gamma$, the deviations become smaller since Coulomb effects dominate the behavior at small distances.

Let us now turn to the dynamical properties. In case of an OCP, charge and mass fluctuations are identical because of the rigid opposite charge background. In our simulations, we have calculated the density-density correlation function (5) and, by numerical Fourier transformation, obtained the dynamical structure factor $S(q, \omega)$ for several (from 6 to 10, depending on the simulation) wave numbers, the values of which are determined by the size of the simulation box L (see above). The value of the smallest wave number is given in Table I. The frequency dependence of $S(q, \omega)$ for several wave vectors is presented in Figs. 2-4 for the Coulomb and Kelbg potentials. Also, the results of the mean-field models are shown. The peak of the structure factor is related to the optical plasmon (Langmuir mode) of the electrons, its position shows the plasmon frequency $\Omega(k)$, its width - the damping of the mode. In the limit $k \rightarrow 0, \Omega(k) \rightarrow \omega_{p l}$ for all models. For increasing wave numbers, the width of the peak grows steadily, and it merges with the continuum of single-particle excitations, e.g. [13,14, therefore, no results for larger wave numbers are shown.

Consider now the results for the plasmon dispersion more in detail, cf. Fig. 5. First, we discuss the mean field results (4) which are calculated using the Vlasov and RPA polarizations, Eqs. (2) and (3), respectively. The Vlasov result was computed using the formulas given in the review of Kugler [24], and for the RPA, a code was developed which accurately evaluates the pole integration in Eq. (3). Both approximations show the same general trend for small and intermediate wave numbers: with increasing wave number, the plasmon frequency and the damping increase. At large $q$, the dispersion exhibits a maximum and decreases again. In all situations, the RPA yields a slightly smaller frequency than the Vlasov 
result, whereas the damping values are very close to each other.

Let us now turn to the simulation results. The Coulomb and Kelbg simulations have been performed for exactly the same parameters, except for $\mathrm{N}$ and run time (cf. Table I). (Notice that, in contrast to the Kelbg case, the Coulomb simulations depend only on $\Gamma$ which can be achieved by various combinations of density and temperature). Comparison of the two simulations shows, cf. Fig. 2, that the results for the structure factors are very similar in case of small $\Gamma$. Peak positions and widths as well as the low and high frequency tails are very close to each other. The reason is obvious: since the potentials (and pair distributions, cf. Fig. 1) differ only at a small interparticle distances of the order of $\Lambda$, differences in the structure factor would show up only at $k>2 \pi / \Lambda$, which is about an order of magnitude larger than the wave numbers shown in Fig. 2. There, the plasmon peak has already a width of the order of the frequency and no longer describes a well-defined collective excitation.

It is now interesting to compare the simulation results to the theoretical approximations. The first observation is that the simulation peaks are significantly broader, cf. Fig. 2. This is obvious since the simulations fully include interparticle correlations missing in the meanfield results. Consequently, the plasmon damping contains collisional damping in addition to the Landau damping (which is the only damping mechanism in the mean-field models). Correspondingly, the plasmon peaks in the simulations are shifted to smaller frequencies. This effect grows with increasing wave number as well as with increasing coupling (see also Fig. 5). We note that, in our simulations, this shift is observed for all wave numbers, which is in contrast to the result of Hansen [see Fig. 9 of Ref. [23] for $q=0.6187$ ]. In other words, the plasmon dispersion curves from the MD simulations are lower than the mean-field result for all wave vectors $\vec{k}$, which is seen more clearly in Fig. 5. As expected the MD curves for the structure factor are much closer to the RPA than to the Vlasov result.

In Fig. 5 we plot the optical plasmon dispersion curves for three values of the coupling parameter for the Vlasov and RPA dispersions together with the simulation results. We further show the well-known analytical approximation to the Langmuir dispersion, 


$$
\omega(q)=\omega_{p l}\left(1+\frac{q^{2}}{\Gamma}\right)^{1 / 2}
$$

Clearly, this predicts a monotonically increasing dispersion. However, this approximation is valid only for $k<1 / r_{D}$ and for $\Gamma<1$. Let us now consider the simulation results which do not have this limitation. In Fig. 5 we show the MD results for a Coulomb potential and for the Kelbg potential for three values of the degeneracy parameter, $\rho \Lambda^{3}=0.1,0.5,1.0$. One clearly sees that, for these parameters, the dispersion is positive, $d \omega(q) / d q>0$, up to wave numbers of the order of one over the mean interparticle distance. For larger $q$, the dispersion changes sign. This general trend is observed for the Coulomb and the Kelbg potential. On the other hand, with increasing quantum effects, $\rho \Lambda^{3}$, the deviations between the two potentials are growing, which becomes more pronounced as $\Gamma$ increases, cf. the curves for $\Gamma=1$ and $\Gamma=4$ : the dispersion in case of the Kelbg potential shows a softer increase with increasing wave number and reaches a lower maximum approximately at the same wave number as in the Coulomb case. We mention that this sign change of the dispersion has not been reported by Hansen [可. Comparing the simulations with the mean-field results, we again see that the MD dispersions proceed lower than the mean field results, and this effect grows with increasing $\Gamma$ and increasing wave number. Once more, we confirm that the RPA dispersion is much closer to the MD result than the Vlasov dispersion, at least for $\Gamma \leq 0.5$. [As mentioned above, the simulation results for the dispersion show certain statistical fluctuations due to the varying accuracy of the results for the different wave numbers].

Let us now consider the plasmon damping more in detail. Fig. 6, shows the damping (full width at half maximum of the plasmon peak of the structure factor) as a function of wave number. It is interesting to compare with the familiar analytical expression from the Vlasov theory, e.g. [26],

$$
\delta(\kappa)=\sqrt{\frac{\pi}{8}} \frac{\sqrt{1+3 \kappa^{2}}}{\kappa^{3}} e^{-\frac{1}{2 \kappa^{2}}-\frac{3}{2}}
$$

where $\kappa \equiv k r_{D}$ is the dimensionless wave number in units of the inverse the Debye radius $r_{D}$ given in Table I. Formula (14) is derived under the condition that the damping is much 
smaller then the frequency $[\delta(q) \ll \omega(q)]$, and is limited to small wave numbers $\kappa \ll 1$. As expected, the damping given by formula (14) which is only Landau damping, is much smaller than the damping found in the simulations, as the latter contain the full collisional damping also. Obviously, for small coupling and small q, Eq. (14) shows the correct trend. However, deviations increase rapidly with growing coupling parameter. Furthermore, the simulations which are not limited to small wave numbers, show a qualitatively different behavior at large $q$ : a monotonic increase of the damping. Interesingly, with increasing $\Gamma$ the damping is reduced, cf. Figs. 6a,b.

Finally, we try to extend the analytical result for the plasmon dispersion, Eq. (13), to larger $\Gamma$ and to include quantum effects. To this end, we used the MD data with the Kelbg

potential to construct an improved fit of the form $\omega(q)=\omega_{p l}\left(1+a q^{2}+b q^{4}\right)^{1 / 2}$. The result is shown in Fig. 7 for $\Gamma=1$ and $\Gamma=4$. Due to the large fluctuations in the dispersion data and the increasing damping for large wave numbers, we used a weighted fit where the smallest $q$-values had the largest weight and the statistical errors of the individual points have been taken into account. Table II contains the resulting fit parameters. According to this data both parameters $a$ and $b$ depend on $\Gamma$ and $\rho \Lambda^{3}$. The parameter $a$ is close to $1 / \Gamma$ in agreement with Eq. (13), but with increasing $\Gamma$, deviations are growing, compare Table II. We see no systematic influence of quantum effects on the parameter $a$ for $\Gamma=1$. Noticeable effects show up for $\Gamma=4$, where increased degeneracy leads to a reduction of the coefficient a. The second fit parameter allows to qualitatively reproduce the change of the sign of the dispersion. The overall agreement is satisfactory for wave numbers up to the inverse mean interparticle distance up to which the plasmons are comparatively weakly damped.

\section{DISCUSSION}

We have presented classical molecular dynamics simulations of the dielectric properties of a one-component plasma at intermediate coupling and degeneracy, $\Gamma \leq 4$ and $\rho \Lambda^{3} \leq 1$. While classical MD simulations can be extended to very large values of $\Gamma$, they have lim- 
ited applicablility to quantum plasmas. We used, as an effective quantum pair potential, the Kelbg potential which correctly describes quantum diffraction effects for small $\Gamma$. In general, we found that the simulation results for the dielectric properties and the plasmon dispersion with the Coulomb and the Kelbg potential are rather close, but start to deviate from each other as $\Gamma$ increases. Nevertheless, the use of the Kelbg potential is preferable. It correctly treats close collisions, i.e. the two-particle interaction at distances smaller than the DeBroglie wavelength. This is of even higher importance in the case of two-component plasmas where the Kelbg potential allows to avoid the collapse of oppositely charged particles. Therefore, the present investigation should be important for future work on two-component plasmas. Finally, we mention that the Kelbg potential is only the first term of a $\Gamma$ expansion. Therefore, for $\Gamma>1$ the account of higher order corrections to the quantum diffraction effects is necessary. Work on this subject is in progress. 


\section{REFERENCES}

[1] J.P. Hansen, Phys. Rev. A 8, 3096 (1973)

[2] J.M. Caillol, D. Levesque, J.J. Weis, and J.P. Hansen, J. Stat. Phys 28, 325 (1982)

[3] J. Ortner, F. Schautz, and W. Ebeling, Phys. Rev. E 56, 1 (1997)

[4] R.K. Moudgil, P.K. Ahluwalia, and K. Tankeshwar, Phys. Rev. B 54, 8809 (1996)

[5] W. Schülke, K. Höppner, and A. Kaprolat, Phys. Rev. B 54, 17464 (1996)

[6] S.G. Brush, H.L. Sahlin, and E. Teller, J. Chem. Phys. 45, 2102 (1966)

[7] W.L. Slattery, E.D. Dollen, and H.E.DeWitt, Phys. Rev. A 26, 2255 (1982)

[8] S. Ogata, S. Ichimaru, Phys. Rev. A 36, 5451 (1987)

[9] R.T. Farouki, and S. Hamaguchi, Phys. Rev. E 47, 4330 (1993)

[10] S. Ichimaru, "Statistical Plasma Physics" Vol.II, Addison-Wesley Publishing Company, 1994; G.S. Stringfellow, H.E. DeWitt, and W.L. Slattery, Phys. Rev. A 41, 1105 (1990)

[11] D. Pines, and Ph. Nozieres, "The Theory of Quantum Liquids", Benjamin, New York 1966

[12] G.D. Mahan, "Many-Particles Physics", Plenum Press, New York/ London 1990

[13] W.D. Kraeft, D. Kremp, W. Ebeling, and G. Röpke, "Quantum Statistics of Charged Particle Systems" (Plenum, London, New York, 1986)

[14] M. Bonitz, "Quantum Kinetic Theory", B.G. Teubner, Stuttgart/Leipzig 1998

[15] N. Kwong, and M. Bonitz, Phys. Rev. Lett. 84, 1768 (2000)

[16] See e.g. D. Klakow, C. Toepffer, and P.-G. Reinhard, Phys. Lett. A 192, 55 (1994); V.S. Filinov, J. Mol. Phys. 88, 1517, 1529 (1996)

[17] G. Kelbg, Ann. Physik (Leipzig) 12, 219 (1963); 13, 354 (1964); 14, 394 (1964) 
[18] W.C. Swope, H.C. Andersen, P.H. Berens, and K.R. Wilson, J. Chem. Phys. 76, 637, (1982)

[19] W. Ebeling, H.J. Hoffmann, and G. Kelbg, Contr. Plasma Phys. 7, 233 (1967)

[20] B.R.A. Nijboer, and F.W. De Wette, Physica XXIII, 309 (1957)

[21] M.J.L. Sangster, and M. Dixon, Advances in Physics 25, 247 (1976)

[22] C. Deutsch, Phys. Lett. A 60, 317 (1977)

[23] J.P. Hansen, I.R. McDonald, and E.L. Pollock, Phys. Rev. A 11, 1025 (1975)

[24] A.A. Kugler, J. Stat. Phys. 8, 107 (1973)

[25] G. Zwicknagel, PhD thesis, University of Erlangen 1994

[26] A.F. Aleksandrov, L.S. Bogdankievich, A.A. Rukhadze, "Principles of Plasma Electrodynamics“, Springer, 1984 


\section{TABLES}

TABLE I. Parameters of the molecular dynamics simulations with the Kelbg potential. Numbers in parentheses refer the runs with Coulomb potential.

\begin{tabular}{|c|c|c|c|c|c|c|c|c|}
\hline \hline$\Gamma$ & $\rho \Lambda^{3}$ & $\rho,\left[\mathrm{cm}^{-3}\right]$ & $\mathrm{T},[\mathrm{K}]$ & $\omega_{p l},[\mathrm{fs}]^{-1}$ & $r_{D} / \bar{r}$ & $N$ & $k_{m i n} \bar{r}$ & run time, $\left[T_{p l}\right]$ \\
\hline 0.5 & 0.1 & $9.12 \cdot 10^{21}$ & $1.126 \cdot 10^{5}$ & 5.387 & 0.816 & $500(250)$ & $0.491(0.619)$ & $515(341)$ \\
0.5 & 0.5 & $2.28 \cdot 10^{23}$ & $3.292 \cdot 10^{5}$ & 26.940 & & $400(250)$ & $0.529(0.619)$ & $429(429)$ \\
\hline 1.0 & 0.1 & $1.14 \cdot 10^{21}$ & $2.228 \cdot 10^{4}$ & 1.905 & 0.577 & 250 & 0.619 & $290(327)$ \\
1.0 & 0.5 & $2.85 \cdot 10^{22}$ & $8.23 \cdot 10^{4}$ & 9.524 & & 250 & 0.619 & $682(682)$ \\
1.0 & 1.0 & $1.14 \cdot 10^{23}$ & $1.31 \cdot 10^{5}$ & 19.048 & & 250 & 0.619 & 477 \\
\hline 4.0 & 0.1 & $1.78 \cdot 10^{19}$ & $1.76 \cdot 10^{3}$ & 0.238 & 0.289 & 250 & 0.619 & $570(227)$ \\
4.0 & 1.0 & $1.78 \cdot 10^{21}$ & $8.17 \cdot 10^{3}$ & 2.381 & & 250 & 0.619 & 716 \\
\hline \hline
\end{tabular}

TABLE II. Fit parameters of the Langmuir dispersion curves shown on Fig. 7. The fit equation was taken in the form of $\omega(q) / \omega_{p l}=\left(1+a q^{2}+b q^{4}\right)^{1 / 2}$. Parameters of the fit for $\Gamma=1$ and $\rho \Lambda^{3}=$ 0.1 are less reliable, because of the absence of data for big wave vectors, cf. Table I.

\begin{tabular}{|c|c|c|c|}
\hline \hline$\Gamma$ & $\rho \Lambda^{3}$ & $\mathrm{a}$ & $\mathrm{b}$ \\
\hline 1.0 & 0.1 & $1.013 \pm 0.031$ & $-0.260 \pm 0.023$ \\
1.0 & 0.5 & $1.074 \pm 0.041$ & $-0.288 \pm 0.013$ \\
1.0 & 1.0 & $0.975 \pm 0.055$ & $-0.259 \pm 0.018$ \\
\hline 4.0 & 0.1 & $0.169 \pm 0.015$ & $-0.034 \pm 0.006$ \\
4.0 & 1.0 & $0.121 \pm 0.007$ & $-0.025 \pm 0.003$ \\
\hline \hline
\end{tabular}




\section{FIGURES}

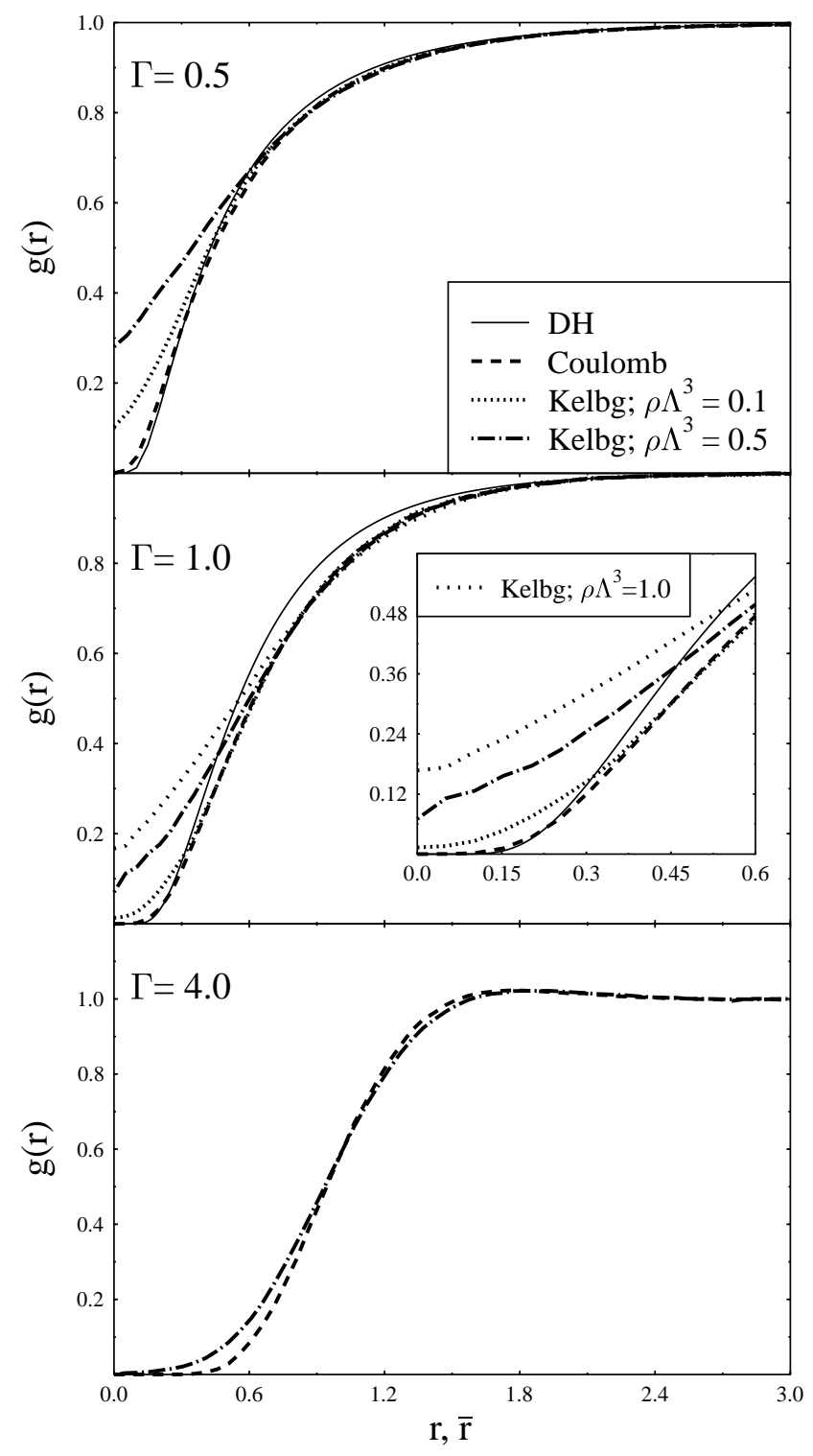

FIG. 1. Pair distribution functions for $\Gamma=0.5$ (upper figure), $\Gamma=1.0$ (middle figure), 4.0 (lower figure), and $\rho \Lambda^{3}=0.1,0.5,1.0$ for systems with Coulomb and Kelbg potential. Further, the Debye-Hückel (DH) limit is shown (solid line). Line styles are the same in all three figures. Inset in the middle Fig. shows the influence of the degeneracy at small distances. The result for $\Gamma=$ $4.0, \rho \Lambda^{3}=0.1$ with Kelbg potential are not distinguishable from the Coulomb result and are not plotted. 


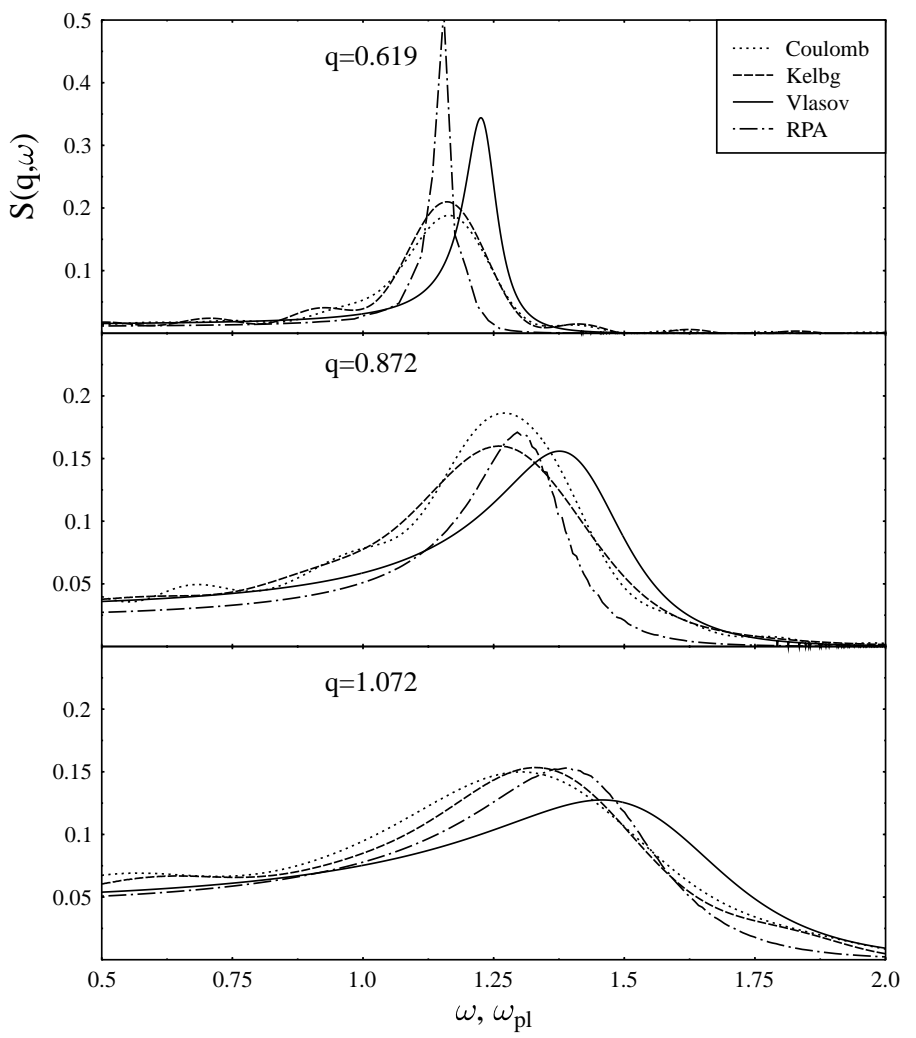

FIG. 2. Dynamical structure factor for an OCP at $\Gamma=1$ and $\rho \Lambda^{3}=0.1$ from MD simulations with Coulomb and Kelbg potentials. In addition, Vlasov and RPA results are shown. The wave numbers are shown in the figures in units of $\bar{r}$, i.e. $q=k \bar{r}$. 


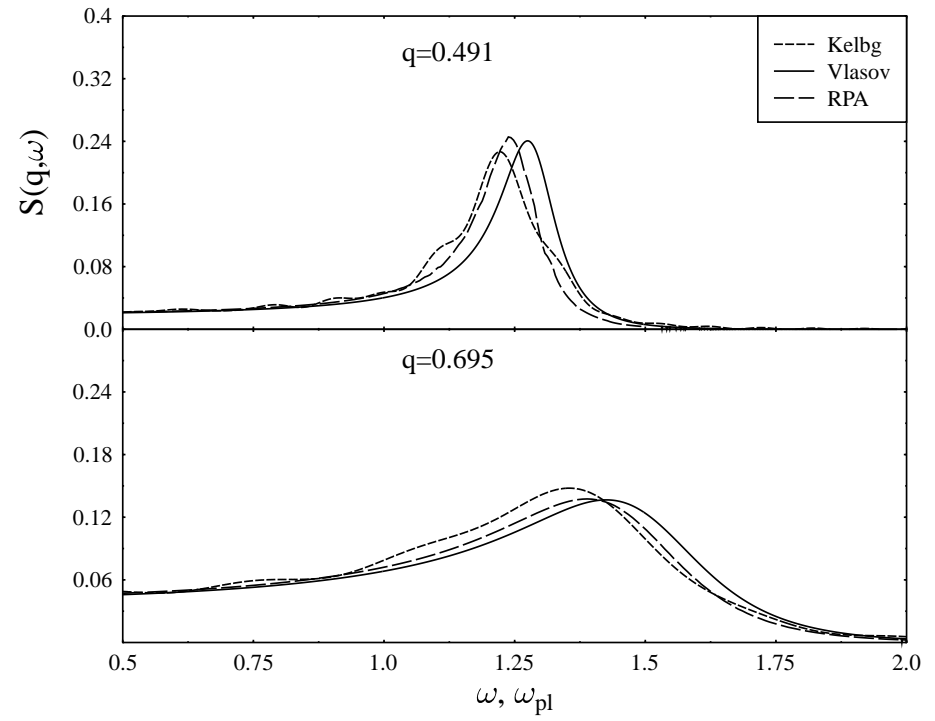

FIG. 3. Same as Fig. 2, but for $\Gamma=0.5$ and $\rho \Lambda^{3}=0.1$. The values of the wave numbers differ from Fig. 1 due to the different particle numbers, cf. Table I.

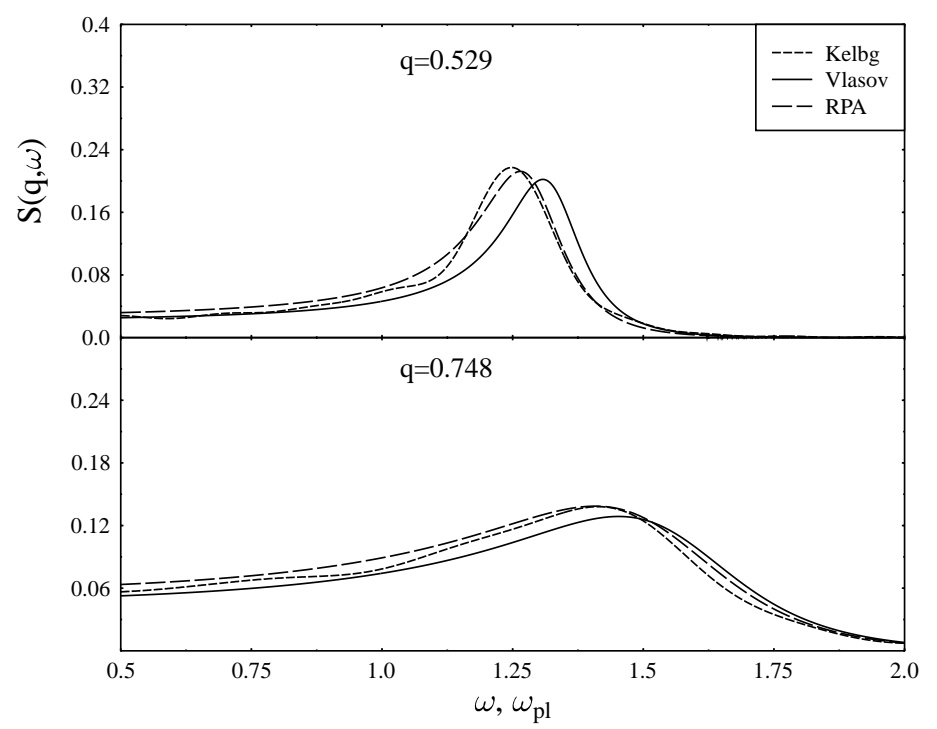

FIG. 4. Same as Fig. 3, but for $\rho \Lambda^{3}=0.5$. 

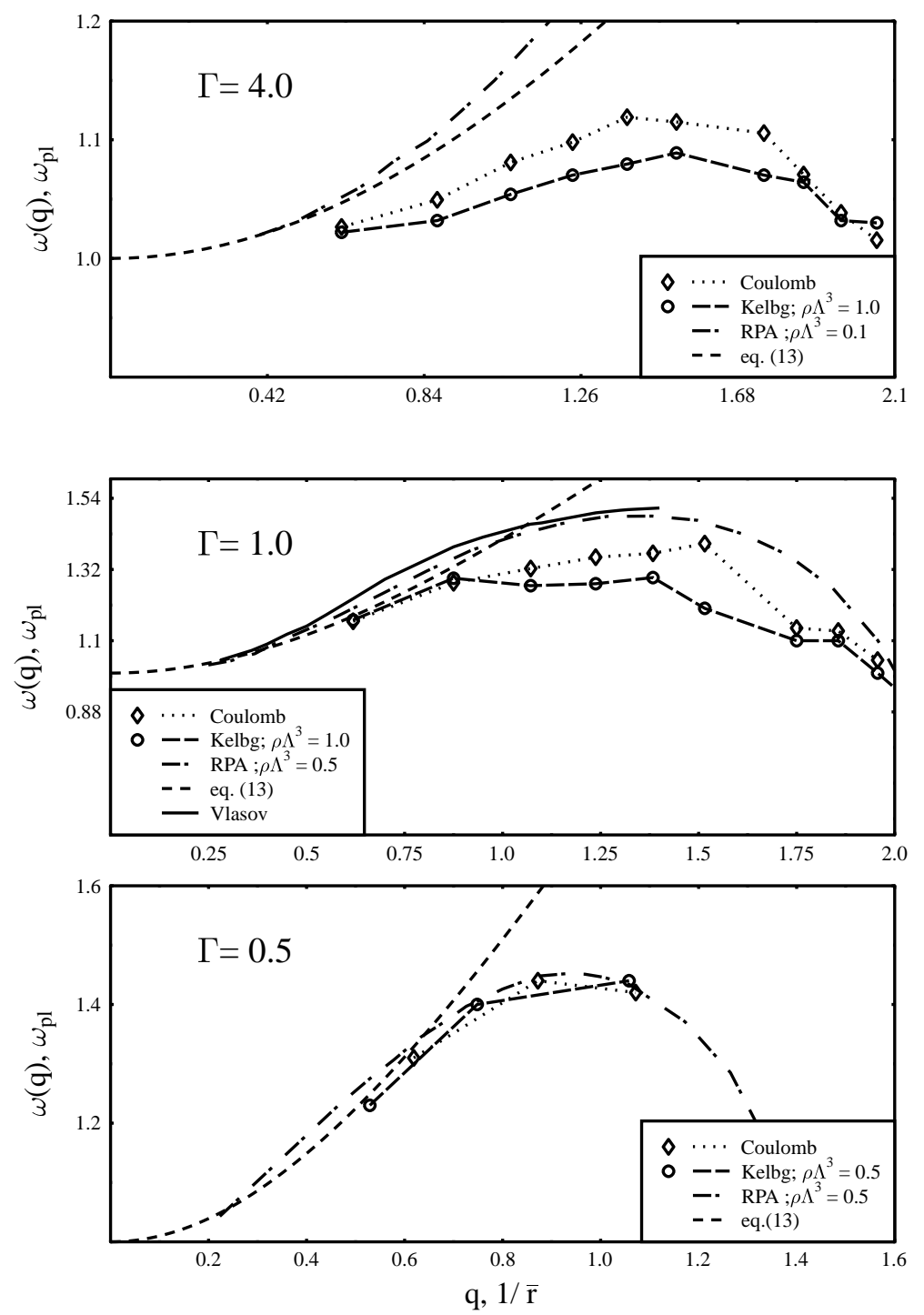

FIG. 5. Optical plasmon dispersion for various coupling and degeneracy parameters from MD simulations with Coulomb and quantum potentials. Also shown are results of the Vlasov and RPA approximations, and of the analytical approximation of Eq. (13). For $\Gamma=4.0$ and $\rho \Lambda^{3}=0.1$ (upper graph) the MD simulations with Kelbg potential and the RPA curve are not shown since they almost coincide with the Coulomb simulation and the Vlasov curve, respectively. 

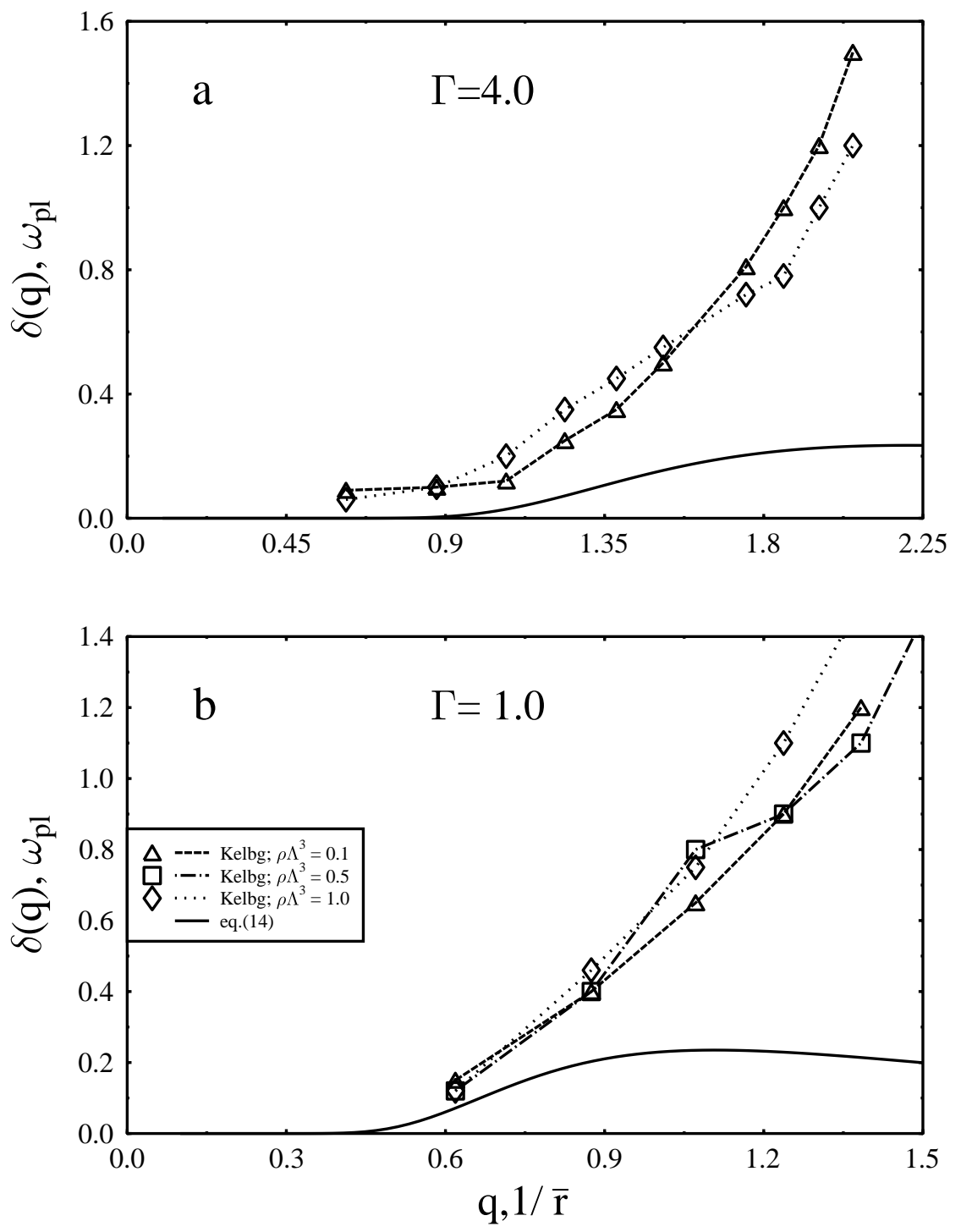

FIG. 6. Damping of Langmuir waves from MD simulations with the Kelbg potential for various values of $\Gamma$ and $\rho \Lambda^{3}$. Solid lines are the analytical small damping limit of the Vlasov theory, Eq. (14). 

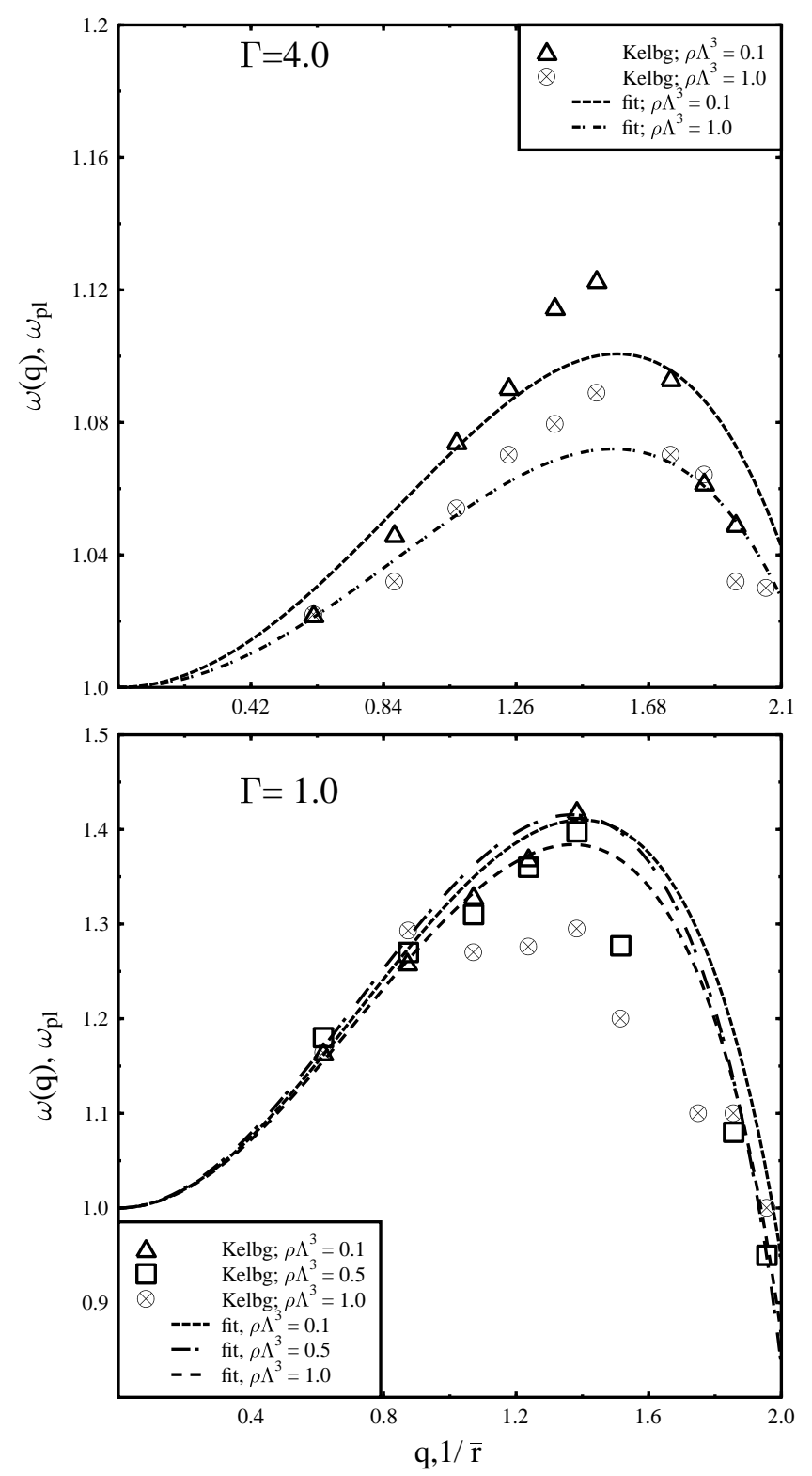

FIG. 7. Dispersion of Langmuir oscillations from MD simulations with the Kelbg potential for various values of the coupling and degeneracy. Symbols are MD results, lines the best fits to the low wave number part $(q<1 / \bar{r})$, the fit formula and parameters are given in Table II. 